\title{
ROS1 Rearrangement Analysis Was Not Performed
}

National Cancer Institute

\section{Source}

National Cancer Institute. ROS1 Rearrangement Analysis Was Not Performed. NCI

Thesaurus. Code C160496.

An indication that ROS1 rearrangement analysis was not performed during the study. 